TRANSACTIONS OF THE

AMERICAN MATHEMATICAL SOCIETY

Volume 364, Number 8, August 2012, Pages 4303-4322

S 0002-9947(2012)05640-6

Article electronically published on March 22, 2012

\title{
ON THE BEST CONSTANTS \\ IN THE WEAK TYPE INEQUALITIES \\ FOR RE-EXPANSION OPERATOR AND HILBERT TRANSFORM
}

\author{
ADAM OSȨKOWSKI
}

\begin{abstract}
We study the weak type inequalities for the operator $I-\mathcal{F}_{s} \mathcal{F}_{c}$, where $\mathcal{F}_{c}$ and $\mathcal{F}_{s}$ are the cosine and sine Fourier transforms on the positive half line, respectively, and $I$ is the identity operator. We also derive sharp constants in related weak type estimates for $I-\mathcal{H}^{\mathbb{T}}, I-\mathcal{H}^{\mathbb{R}}$ and $I-\mathcal{H}^{\mathbb{R}}$, where $\mathcal{H}^{\mathbb{T}}, \mathcal{H}^{\mathbb{R}}$ and $\mathcal{H}^{\mathbb{R}}$ denote the Hilbert transforms on the circle, on the real line and the positive half-line, respectively. Our main tool is the weak type inequality for orthogonal martingales, which is of independent interest.
\end{abstract}

\section{INTRODUCTION}

The motivation for the results of this paper comes from the question about the weak norms of the re-expansion operator on $\mathbb{R}_{+}=(0, \infty)$. Let us start with introducing the necessary background and notation. Let $\mathcal{F}_{c}$ and $\mathcal{F}_{s}$ be the cosine and sine Fourier transforms on $\mathbb{R}_{+}$, respectively. That is, for $x>0$,

$$
\mathcal{F}_{c} f(x)=\sqrt{\frac{2}{\pi}} \int_{\mathbb{R}_{+}} f(t) \cos t x \mathrm{~d} t, \quad \mathcal{F}_{s} f(x)=\sqrt{\frac{2}{\pi}} \int_{\mathbb{R}_{+}} f(t) \sin t x \mathrm{~d} t .
$$

Both $\mathcal{F}_{c}$ and $\mathcal{F}_{s}$ are unitary and self-adjoint operators on $L^{2}\left(\mathbb{R}_{+}\right)$. The re-expansion operator is defined by $\Pi=\mathcal{F}_{s} \mathcal{F}_{c}$. It is interesting from the analytical point of view as the object of spectral analysis and it also appears naturally in scattering theory. To be more specific, let $\mathcal{H}, \mathcal{H}_{0}$ be two self-adjoint operators on a Hilbert space. Then the wave operators $W_{ \pm}=W_{ \pm}\left(\mathcal{H}, \mathcal{H}_{0}\right)$ are defined by

$$
W_{ \pm}\left(\mathcal{H}, \mathcal{H}_{0}\right)=\lim _{t \rightarrow \pm \infty} e^{i t \mathcal{H}} e^{-i t \mathcal{H}_{0}}
$$

(the limit is understood in the sense of strong operator convergence). One expands a given function with respect to the eigenfunctions of $\mathcal{H}_{0}$ and then takes the inverse transform using the eigenfunctions of $\mathcal{H}$. If we put $\mathcal{H}_{0}, \mathcal{H}$ to be the operator $-\frac{d^{2}}{d x^{2}}$ on $L^{2}\left(\mathbb{R}_{+}\right)$with the boundary conditions $f^{\prime}(0)=0$ and $f(0)=0$, respectively, then $W_{ \pm}\left(\mathcal{H}_{0}, \mathcal{H}\right)= \pm \Pi$ (see Birman [2]). The re-expansion operator also appears in the polar decomposition of $-i \frac{d}{d x}$ on $L^{2}\left(\mathbb{R}_{+}\right)$with the domain defined by $f(0)=0$ (again, see [2]) and arises in other problems of mathematical physics.

Received by the editors February 22, 2011.

2010 Mathematics Subject Classification. Primary 42B10,60G44; Secondary 46E30.

Key words and phrases. Martingale, re-expansion operator, Fourier transform.

The author was partially supported by MNiSW Grant N N201 364436.

(C)2012 American Mathematical Society Reverts to public domain 28 years from publication 
The re-expansion operator can be represented as singular integral operator: for $x>0$,

$$
\Pi f(x)=\frac{1}{\pi} \text { p.v. } \int_{\mathbb{R}_{+}} \frac{2 x f(t)}{x^{2}-t^{2}} \mathrm{~d} t .
$$

It is closely related to $\mathcal{H}_{+}^{\mathbb{R}}$, the Hilbert transform on $\mathbb{R}_{+}$, and $\mathcal{L}$, the Laplace transform, which are given by

$$
\mathcal{H}^{\mathbb{R}_{+}} f(x)=\frac{1}{\pi} \text { p.v. } \int_{\mathbb{R}_{+}} \frac{f(t)}{x-t} \mathrm{~d} t, \quad \mathcal{L} f(x)=\sqrt{\frac{2}{\pi}} \int_{\mathbb{R}_{+}} f(t) e^{-t x} \mathrm{~d} t, \quad x>0 .
$$

The connection is given by the identity $\Pi=\mathcal{H}^{\mathbb{R}_{+}}+\mathcal{H}_{1}$, where

$$
\mathcal{H}_{1} f(x)=\frac{1}{2} \mathcal{L}^{2} f(x)=\frac{1}{\pi} \int_{\mathbb{R}_{+}} \frac{f(t)}{x+t} \mathrm{~d} t, \quad x>0 .
$$

In what follows, we will also need the Hilbert transforms $\mathcal{H}^{\mathbb{R}}, \mathcal{H}^{\mathbb{T}}$ on the real line and on the unit circle, respectively. These operators are given by

$$
\mathcal{H}^{\mathbb{R}} f(x)=\frac{1}{\pi} \text { p.v. } \int_{\mathbb{R}} \frac{f(t)}{x-t} \mathrm{~d} t, \quad \mathcal{H}^{\mathbb{T}} f(x)=\frac{1}{2 \pi} \int_{-\pi}^{\pi} f(x) \cot \frac{x-t}{2} \mathrm{~d} t .
$$

Our main interest will be in calculating norms of various operators related to $\Pi$. Clearly, we have $\|\Pi\|_{L^{2}\left(\mathbb{R}_{+}\right) \rightarrow L^{2}\left(\mathbb{R}_{+}\right)}=1$. It can be easily shown that $\Pi$ can be extended to a bounded operator on $L^{p}\left(\mathbb{R}_{+}\right)$for $1<p<\infty$. In fact, as proved by Hollenbeck et al. 8 , we have

$$
\|\Pi\|_{L^{p}\left(\mathbb{R}_{+}\right) \rightarrow L^{p}\left(\mathbb{R}_{+}\right)}=\cot \frac{\pi}{2 p^{*}}, \quad p^{*}=\max \{p, p /(p-1)\} .
$$

We see that the expression on the right is precisely the $L^{p}$-norm of the Hilbert transforms $\mathcal{H}^{\mathbb{R}_{+}}, \mathcal{H}^{\mathbb{R}}$ and $\mathcal{H}^{\mathbb{T}}$ : see Gohberg and Krupnik [7] for $p=2^{n}, n=2,3, \ldots$, and for remaining values of $p$, consult Pichorides [11] and Cole (unpublished; see [6]). It will be clear from the reasoning presented in this paper that if $1 \leq p \leq 2$, then the weak $p$-th norms of $\Pi$ and the Hilbert transform also coincide. Therefore, by the results of Davis [3] and Janakiraman [10, we have

$$
\|\Pi\|_{L^{p}\left(\mathbb{R}_{+}\right) \rightarrow L^{p, \infty}\left(\mathbb{R}_{+}\right)}=\left(\frac{1}{\pi} \int_{-\infty}^{\infty} \frac{\left|\frac{2}{\pi} \log \right| t||^{p}}{t^{2}+1} \mathrm{~d} t\right)^{-1 / p}, \quad 1 \leq p \leq 2 .
$$

It is also of interest to investigate the properties of the operator $I-\Pi$; see Birman [2]. A straightforward computation gives

$$
\|I-\Pi\|_{L^{2}\left(\mathbb{R}_{+}\right) \rightarrow L^{2}\left(\mathbb{R}_{+}\right)}=\left\|\mathcal{F}_{c}-\mathcal{F}_{s}\right\|_{L^{2}\left(\mathbb{R}_{+}\right) \rightarrow L^{2}\left(\mathbb{R}_{+}\right)}=\sqrt{2} .
$$

The question about the $L^{p}$-norms of $I-\Pi$ in the case when $p \neq 2$ turns out to be much more difficult. It was answered by Hollenbeck et al. [8]: for $1<p<\infty$ we have

$$
\|I-\Pi\|_{L^{p}\left(\mathbb{R}_{+}\right) \rightarrow L^{p}\left(\mathbb{R}_{+}\right)}=\sqrt{2} \max _{0 \leq \theta \leq 2 \pi}\left[\frac{\left|\cos \left(\theta-\frac{\pi}{4}\right)\right|^{p}+\left|\cos \left(\theta-\frac{\pi}{4}+\frac{\pi}{p}\right)\right|^{p}}{|\cos \theta|^{p}+\left|\cos \left(\theta+\frac{\pi}{p}\right)\right|^{p}}\right]^{1 / p} .
$$

One of our main results will be to derive the weak $L^{p}$-norms of $I-\Pi$ for $1 \leq p \leq 2$. In fact, we will study this problem in a much wider setting. Throughout the paper, $\mathcal{C}$ will denote the class of even, convex functions $\Phi$ on $\mathbb{R}$ satisfying $\Phi(0)=0$ and

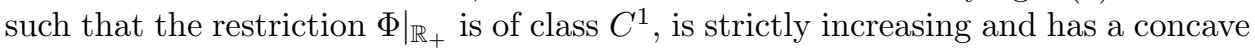


derivative (examples, $\Phi(t)=|t|^{p}$ for $1 \leq p \leq 2 ; \Phi(t)=|t| \log (|t|+1)$ ). For $\Phi \in \mathcal{C}$, we define

$$
K_{\Phi}=\left(\int_{\mathbb{R}} \frac{\Phi(|s+1|)}{\cosh \left(\frac{\pi s}{2}\right)} \mathrm{d} s\right)^{-1} .
$$

Theorem 1.1. Let $\Phi$ be a fixed element of $\mathcal{C}$ and let $f$ be a locally integrable function on $\mathbb{R}_{+}$. Then we have

$$
|\{x \geq 0:|(I-\Pi) f(x)| \geq 1\}| \leq K_{\Phi} \int_{\mathbb{R}_{+}} \Phi(2 f(x)) d x .
$$

The constant $K_{\Phi}$ is the best possible.

In the particular case when $\Phi(t)=|t|^{p}, 1 \leq p \leq 2$, we get the following.

Corollary 1.2. We have

$$
\| I-\left.\Pi\right|_{L^{p}\left(\mathbb{R}_{+}\right) \rightarrow L^{p, \infty}\left(\mathbb{R}_{+}\right)}=2\left(\int_{\mathbb{R}} \frac{|s+1|^{p}}{\cosh \left(\frac{\pi s}{2}\right)} d s\right)^{-1 / p} .
$$

Our second result will be to show that inequality (1.1) carries over to Hilbert transforms with no change in the constant. Let us state this as a separate theorem.

Theorem 1.3. Let $\Phi$ be a fixed function belonging to the class $\mathcal{C}$.

(i) If $f$ is a locally integrable function on $[-\pi, \pi]$, then

$$
\left|\left\{x \in[-\pi, \pi]:\left|\left(I-\mathcal{H}^{\mathbb{T}}\right) f(x)\right| \geq 1\right\}\right| \leq K_{\Phi} \int_{[-\pi, \pi]} \Phi(2 f(x)) d x .
$$

(ii) If $f$ is a locally integrable function on $\mathbb{R}$, then

$$
\left|\left\{x \in \mathbb{R}:\left|\left(I-\mathcal{H}^{\mathbb{R}}\right) f(x)\right| \geq 1\right\}\right| \leq K_{\Phi} \int_{\mathbb{R}} \Phi(2 f(x)) d x .
$$

(iii) If $f$ is a locally integrable function on $\mathbb{R}_{+}$, then

$$
\left|\left\{x \geq 0:\left|\left(I-\mathcal{H}^{\mathbb{R}_{+}}\right) f(x)\right| \geq 1\right\}\right| \leq K_{\Phi} \int_{\mathbb{R}_{+}} \Phi(2 f(x)) d x .
$$

All the inequalities are sharp.

Both theorems above will be established by means of probabilistic tools. Let $(\Omega, \mathcal{G}, \mathbb{P})$ be a complete probability space, filtered by $\left(\mathcal{G}_{t}\right)_{t \geq 0}$, a nondecreasing family of sub- $\sigma$-algebras of $\mathcal{G}$. Let us assume in addition, that $\mathcal{G}_{0}$ contains all the events of probability 0 . Let $X=\left(X_{t}\right)_{t \geq 0}, Y=\left(Y_{t}\right)_{t \geq 0}$ be two adapted real-valued martingales with continuous paths and let $[X, Y]$ denote their quadratic covariance process (see e.g. Dellacherie and Meyer [4 for details). We say that the processes $X$ and $Y$ are orthogonal, if $[X, Y]$ is constant almost surely. Following Bañuelos and Wang [1] and Wang [12, we say that $Y$ is differentially subordinate to $X$, if the process $\left([X, X]_{t}-[Y, Y]_{t}\right)_{t \geq 0}$ is nondecreasing and nonnegative as a function of $t$. For example, let $B$ be a $d$-dimensional Brownian motion starting from 0 and let $H$, $K$ be predictable $\mathbb{R}^{d}$-valued processes satisfying $\left|K_{t}\right| \leq\left|H_{t}\right| \leq 1$ and $\left\langle H_{t}, K_{t}\right\rangle=0$ for all $t>0$ almost surely (here $|\cdot|,\langle\cdot, \cdot\rangle$ denote the standard norm and the scalar product in $\mathbb{R}^{d}$ ). If we set

$$
X_{t}=\int_{0+}^{t} H_{s} \mathrm{~d} B_{s}, \quad Y_{t}=\int_{0+}^{t} K_{s} \mathrm{~d} B_{s}
$$


for $t \geq 0$, then $X$ and $Y$ are orthogonal martingales such that $Y$ is differentially subordinate to $X$. This is an immediate consequence of the identities

$$
[X, Y]_{t}=\int_{0+}^{t}\left\langle H_{s}, K_{s}\right\rangle d s=K_{0} H_{0} B_{0}^{2}
$$

and

$$
[X, X]_{t}-[Y, Y]_{t}=\int_{0+}^{t}\left|H_{s}\right|^{2}-\left|K_{s}\right|^{2} d s .
$$

The probabilistic counterpart of Theorems 1.1 and 1.3 can be stated as follows. Let $Y^{*}$ denote the maximal function of $Y$, given by $\sup _{t \geq 0}\left|Y_{t}\right|$.

Theorem 1.4. Let $\Phi$ be a function from $\mathcal{C}$. Let $X, Y$ be two continuous-path orthogonal martingales such that $Y$ is differentially subordinate to $X$ and $\mathbb{P}\left(X_{0}=-Y_{0}\right)=1$. Then

$$
\mathbb{P}\left(Y^{*} \geq 1\right) \leq K_{\Phi} \sup _{t} \mathbb{E} \Phi\left(X_{t}-Y_{t}\right)
$$

and the constant $K_{\Phi}$ is the best possible.

For related results concerning orthogonal martingales and various applications of these, we refer the interested reader to [1] and [10].

A few words about the organization of the paper. First, in the next section, we study the probabilistic result, Theorem 1.4. Namely, we construct a family of certain special subharmonic functions on $\mathbb{R}^{2}$ which, together with Itô's formula, enable us to establish the estimate (1.5). Then, in Section 3, we show how this martingale inequality leads to (1.2), (1.3) and (1.4). In addition, we show there that the constant $K_{\Phi}$ is optimal in these three estimates and this immediately implies that the inequality (1.5) is also sharp. The final part of the paper is devoted to the proof of Theorem 1.1 .

\section{WEAK TYPE INEQUALITY FOR ORTHOGONAL MARTINGALES}

2.1. On the method of proof. Let $\Phi$ be a given function from $\mathcal{C}$ and let $S$ denote the strip $\mathbb{R} \times(-1,1)$. We will construct a continuous function $U_{\Phi}: \mathbb{R}^{2} \rightarrow \mathbb{R}$ which satisfies the following properties (2.1)-(2.5):

$$
\begin{aligned}
& U_{\Phi} \text { is subharmonic on } \mathbb{R}^{2} \text { and harmonic on } S, \\
& U_{\Phi}(0,0)=K_{\Phi}^{-1}, \\
& U_{\Phi x x}>0 \text { on } S, \\
& U_{\Phi}(x,-x) \geq U_{\Phi}(0,0) \quad \text { for all } x \in \mathbb{R}, \\
& \Phi(x-y) \leq U_{\Phi}(x, y) \leq U_{\Phi}(0,0) 1_{\{|y|<1\}}+\Phi(x-y) \text { for all } x, y \in \mathbb{R} .
\end{aligned}
$$

The existence of such a function implies the inequality

$$
K_{\Phi}^{-1} \sup _{t \geq 0} \mathbb{P}\left(\left|Y_{t}\right| \geq 1\right) \leq \sup _{t \geq 0} \mathbb{E} \Phi\left(X_{t}-Y_{t}\right), \quad t \geq 0,
$$

and this immediately yields (1.5) by a well-known stopping time argument. To see how $U_{\Phi}$ leads to (2.6), fix $t \geq 0$ and note that we may assume that the right-hand side of (2.6) is finite. Introduce the stopping time $\tau=\inf \left\{t:\left|Y_{t}\right| \geq 1\right\}$ and consider 
a two-dimensional process $Z=\left(\left(X_{t}, Y_{t}\right)\right)_{t \geq 0}$. When $t \in(0, \tau], Z$ takes values in $\bar{S}$, where $U_{\Phi}$ is of class $C^{2}$ (see (2.1) $)$. Thus we may apply Itô's formula to obtain

$$
U_{\Phi}\left(Z_{\tau \wedge t}\right)=I_{0}+I_{1}+\frac{I_{2}}{2}
$$

where

$$
\begin{aligned}
& I_{0}=U_{\Phi}\left(Z_{0}\right), \\
& I_{1}=\int_{0+}^{\tau \wedge t} U_{\Phi x}\left(Z_{s}\right) d X_{s}+\int_{0+}^{\tau \wedge t} U_{\Phi y}\left(Z_{s}\right) d Y_{s}, \\
& I_{2}=\int_{0+}^{\tau \wedge t} U_{\Phi x x}\left(Z_{s}\right) d[X, X]_{s}+2 \int_{0+}^{\tau \wedge t} U_{\Phi x y}\left(Z_{s}\right) d[X, Y]_{s}+\int_{0+}^{\tau \wedge t} U_{\Phi y y}\left(Z_{s}\right) d[Y, Y]_{s} .
\end{aligned}
$$

We have that $I_{0}=U_{\Phi}\left(X_{0}, Y_{0}\right) \geq U_{\Phi}(0,0)$, by (2.4) and the assumption $X_{0}=-Y_{0}$. Moreover, $\mathbb{E} I_{1}=0$, by the properties of stochastic integrals. To deal with $I_{2}$, we use the orthogonality of $X$ and $Y$ together with harmonicity of $U_{\Phi}$ on $S$ to obtain

$$
\begin{aligned}
I_{2} & =\int_{0+}^{\tau \wedge t} U_{\Phi x x}\left(Z_{s}\right) d[X, X]_{s}+\int_{0+}^{\tau \wedge t} U_{\Phi y y}\left(Z_{s}\right) d[Y, Y]_{s} \\
& =\int_{0+}^{\tau \wedge t} U_{\Phi x x}\left(Z_{s}\right) d\left([X, X]_{t}-[Y, Y]_{t}\right) .
\end{aligned}
$$

This is nonnegative by virtue of (2.3) and the differential subordination. Both sides of (2.7) are integrable by (2.5) and the assumption $\sup _{t \geq 0} \mathbb{E} \Phi\left(X_{t}-Y_{t}\right)<\infty$; thus, taking expectation gives the bound $\mathbb{E} U_{\Phi}\left(Z_{\tau \wedge t}\right) \geq U_{\Phi}(0,0)$. Now if we apply (2.5), we see that

$$
\begin{aligned}
U_{\Phi}(0,0) & \leq U_{\Phi}(0,0) \mathbb{P}\left(\left|Y_{\tau \wedge t}\right|<1\right)+\mathbb{E} \Phi\left(X_{\tau \wedge t}-Y_{\tau \wedge t}\right) \\
& \leq U_{\Phi}(0,0) \mathbb{P}\left(\left|Y_{t}\right|<1\right)+\mathbb{E} \Phi\left(X_{t}-Y_{t}\right) .
\end{aligned}
$$

By (2.2), this is precisely the desired bound (2.6). Thus, we have reduced the problem of proving this weak type inequality to that of finding an appropriate function $U_{\Phi}$.

2.2. The special functions. Suppose that $\Phi$ is a given function from the class $\mathcal{C}$. First we introduce a certain auxiliary object, a real-valued function $\mathcal{U}_{\Phi}$ on the upper plane $H=\mathbb{R} \times(0, \infty)$. It is defined by the Poisson integral

$$
\mathcal{U}_{\Phi}(\alpha, \beta)=\frac{1}{\pi} \int_{\mathbb{R}} \frac{\beta \Phi\left(\frac{2}{\pi} \log |t|+\operatorname{sgn} t\right)}{(\alpha-t)^{2}+\beta^{2}} \mathrm{~d} t .
$$

Clearly, the function $\mathcal{U}_{\Phi}$ is harmonic on $H$ and satisfies

$$
\lim _{(\alpha, \beta) \rightarrow(t, 0)} \mathcal{U}_{\Phi}(\alpha, \beta)=\Phi\left(\frac{2}{\pi} \log |t|+\operatorname{sgn} t\right),
$$

if $t \neq 0$. Consider a conformal mapping $\varphi$, given by $\varphi(z)=i \exp (\pi z / 2)$, or, in real coordinates,

$$
\varphi(x, y)=\left(-e^{\pi x / 2} \sin (\pi y / 2), e^{\pi x / 2} \cos (\pi y / 2)\right) .
$$

It is easy to check that $\varphi$ maps $S$ onto $H$. Now, the desired function $U_{\Phi}: \mathbb{R}^{2} \rightarrow \mathbb{R}$ is defined by

$$
U_{\Phi}(x, y)= \begin{cases}\mathcal{U}_{\Phi}(\varphi(x, y)) & \text { if }(x, y) \in S \\ \Phi(x-y) & \text { if }(x, y) \notin S\end{cases}
$$


The remainder of this section is devoted to the verification of the conditions (2.1)(2.5). The first three of them are rather easy.

Lemma 2.1. The function $U_{\Phi}$ satisfies (2.1), (2.2) and (2.3).

Proof. To show the first property, note first that $U_{\Phi}$ is continuous on $\mathbb{R}^{2}$; this is a consequence of (2.8). Observe that inside $S$ the function $U_{\Phi}$ is a harmonic lift of a subharmonic function $(x, y) \mapsto \Phi(x-y)$. This immediately yields (2.1).

We turn to the second condition. By the definition of $U_{\Phi}$, we may write

$$
\begin{aligned}
U_{\Phi}(0,0)=\mathcal{U}_{\Phi}(0,1) & =\frac{1}{\pi} \int_{\mathbb{R}} \frac{\Phi\left(\frac{2}{\pi} \log |t|+\operatorname{sgn} t\right)}{t^{2}+1} \mathrm{~d} t \\
& =\frac{1}{\pi} \int_{0}^{\infty} \frac{\Phi\left(\frac{2}{\pi} \log t+1\right)}{t^{2}+1} \mathrm{~d} t+\frac{1}{\pi} \int_{0}^{\infty} \frac{\Phi\left(\frac{2}{\pi} \log t-1\right)}{t^{2}+1} \mathrm{~d} t .
\end{aligned}
$$

Substitute $t:=1 / t$ in the second integral to get

$$
U_{\Phi}(0,0)=\frac{2}{\pi} \int_{0}^{\infty} \frac{\Phi\left(\frac{2}{\pi} \log t+1\right)}{t^{2}+1} \mathrm{~d} t=\int_{\mathbb{R}} \frac{\Phi(s+1)}{\cosh \left(\frac{\pi s}{2}\right)} \mathrm{d} s=K_{\Phi}^{-1},
$$

as desired. Finally, to show (2.3), we derive that

$$
U_{\Phi}(x, y)=\frac{1}{\pi} \int_{\mathbb{R}} \frac{\cos \left(\frac{\pi}{2} y\right) \Phi\left(\frac{2}{\pi} \log |s|+\operatorname{sgn} s+x\right)}{\left(\sin \left(\frac{\pi}{2} y\right)+s\right)^{2}+\cos ^{2}\left(\frac{\pi}{2} y\right)} \mathrm{d} s
$$

for $(x, y) \in S$. This immediately gives the strict convexity of $U_{\Phi}(\cdot, y)$.

The conditions (2.4) and (2.5) turn out to be much more difficult. Fortunately, by Fubini's theorem, they have the following property. Namely, if they are valid for $\Phi$ 's belonging to a certain class, say $\mathcal{K}$, then they also hold for the functions lying in the convex hull of $\mathcal{K}$. Thus, our plan is to establish these two properties for a class of "simple" functions which generates $\mathcal{C}$.

Let $\mathcal{K}=\left\{\Phi_{a}: \mathbb{R} \rightarrow \mathbb{R}: a \in[0, \infty]\right\}$, where, for finite and strictly positive $a$,

$$
\Phi_{a}(t)= \begin{cases}\frac{1}{2} t^{2} & \text { if }|t| \leq a, \\ a|t|-\frac{1}{2} a^{2} & \text { if }|t|>a .\end{cases}
$$

Furthermore, set $\Phi_{0}(t)=|t|$ and $\Phi_{\infty}(t)=\frac{1}{2} t^{2}$ for $t \in \mathbb{R}$. For simplicity, we will use the notation $U_{a}:=U_{\Phi_{a}}, a \in[0, \infty]$. In the particular case when $a=\infty$ we can give a simple formula for this function:

$$
U_{\infty}(x, y)= \begin{cases}\frac{1}{2}\left(x^{2}-y^{2}-2 x y+2\right) & \text { if }|y| \leq 1 \\ \frac{1}{2}(x-y)^{2} & \text { if }|y|>1\end{cases}
$$

In the sequence of lemmas below, we study the properties of the functions from class $\mathcal{K}$.

Lemma 2.2. For any $0<a<\infty$ we have $0 \leq U_{a x x} \leq 1$ on $S$.

Proof. The lower bound has been established in the previous lemma. To show the upper bound, note that

$$
\Phi_{a}^{\prime}(t)=-a 1_{(-\infty,-a]}(t)+t 1_{(-a, a)}(t)+a 1_{[a, \infty)}(t),
$$

so $\Phi_{a}^{\prime}\left(x_{1}\right)-\Phi_{a}^{\prime}\left(x_{2}\right) \leq x_{1}-x_{2}$ for all $x_{1}, x_{2} \in \mathbb{R}$. Let us compute $U_{a x}$ on the strip $S$ : by Fubini's theorem, we are allowed to take the derivative under the integral in (2.9). Hence, by the previous estimate, we have $U_{a x}\left(x_{1}, y\right)-U_{a x}\left(x_{2}, y\right) \leq x_{1}-x_{2}$ for 
all $x_{1}, x_{2} \in \mathbb{R}$ and $y \in(-1,1)$. By symmetry, this implies $\left|U_{a x}\left(x_{1}, y\right)-U_{a x}\left(x_{2}, y\right)\right| \leq$ $\left|x_{1}-x_{2}\right|$ for such $x_{1}, x_{2}$ and $y$; this yields the claim.

Lemma 2.3. For any $a \in[0, \infty]$ and $x, y \in \mathbb{R}$ we have $U_{a}(x, y)=U_{a}(-x,-y)$.

Proof. Clearly, it suffices to show the claim for $(x, y) \in S$. It follows from the substitution $s:=-s$ in (2.9) that

$$
\begin{aligned}
U_{a}(x, y)=\frac{1}{2}[ & \frac{1}{\pi} \int_{\mathbb{R}} \frac{\cos \left(\frac{\pi}{2} y\right) \Phi_{a}\left(\frac{2}{\pi} \log |s|+\operatorname{sgn} s+x\right)}{\left(\sin \left(\frac{\pi}{2} y\right)+s\right)^{2}+\cos ^{2}\left(\frac{\pi}{2} y\right)} \mathrm{d} s \\
& \left.+\frac{1}{\pi} \int_{\mathbb{R}} \frac{\cos \left(\frac{\pi}{2} y\right) \Phi_{a}\left(\frac{2}{\pi} \log |s|-\operatorname{sgn} s+x\right)}{\left(\sin \left(\frac{\pi}{2} y\right)-s\right)^{2}+\cos ^{2}\left(\frac{\pi}{2} y\right)} \mathrm{d} s\right] .
\end{aligned}
$$

It remains to substitute $s:=1 / s$ in both of the integrals above and use the fact that $\Phi_{a}$ is an even function on $\mathbb{R}$.

Lemma 2.4. For any $0<a<\infty$ and $x \in(0,1)$ we have

$$
U_{a x}(x, x) \leq 0 .
$$

Proof. Divide the integral in (2.9) into two, over the negative half-line and the positive half-line, and substitute $s=-e^{\pi r / 2}$ and $s=e^{\pi r / 2}$. We obtain

$$
\begin{aligned}
U_{a}(x, y)= & \frac{1}{2} \int_{\mathbb{R}} \frac{e^{\pi r / 2} \cos \left(\frac{\pi y}{2}\right) \Phi_{a}(r+x+1)}{e^{\pi r}+2 e^{\pi r / 2} \sin \left(\frac{\pi y}{2}\right)+1} \mathrm{~d} r \\
& +\frac{1}{2} \int_{\mathbb{R}} \frac{e^{\pi r / 2} \cos \left(\frac{\pi y}{2}\right) \Phi_{a}(r+x-1)}{e^{\pi r}-2 e^{\pi r / 2} \sin \left(\frac{\pi y}{2}\right)+1} \mathrm{~d} r .
\end{aligned}
$$

Therefore, we see that for a fixed $x \in(0,1)$,

$$
\begin{aligned}
F(a):=U_{a x}(x, x)= & \frac{1}{2} \int_{\mathbb{R}} \frac{e^{\pi r / 2} \cos \left(\frac{\pi x}{2}\right) \Phi_{a}^{\prime}(r+x+1)}{e^{\pi r}+2 e^{\pi r / 2} \sin \left(\frac{\pi x}{2}\right)+1} \mathrm{~d} r \\
& +\frac{1}{2} \int_{\mathbb{R}} \frac{e^{\pi r / 2} \cos \left(\frac{\pi x}{2}\right) \Phi_{a}^{\prime}(r+x-1)}{e^{\pi r}-2 e^{\pi r / 2} \sin \left(\frac{\pi x}{2}\right)+1} \mathrm{~d} r .
\end{aligned}
$$

Since

$$
\frac{e^{\pi r / 2} \cos \left(\frac{\pi x}{2}\right)}{e^{\pi r} \pm 2 e^{\pi r / 2} \sin \left(\frac{\pi x}{2}\right)+1}=\frac{\mathrm{d}}{\mathrm{d} r}\left[\frac{2}{\pi} \arctan \left(\frac{e^{\pi r / 2}}{\cos \left(\frac{\pi x}{2}\right)} \pm \tan \left(\frac{\pi x}{2}\right)\right)\right],
$$

an integration by parts gives

$$
\begin{aligned}
F(a)= & a-\frac{1}{\pi} \int_{-a-x-1}^{a-x-1} \arctan \left(\frac{e^{\pi r / 2}}{\cos \left(\frac{\pi x}{2}\right)}+\tan \left(\frac{\pi x}{2}\right)\right) \mathrm{d} r \\
& -\frac{1}{\pi} \int_{-a-x+1}^{a-x+1} \arctan \left(\frac{e^{\pi r / 2}}{\cos \left(\frac{\pi x}{2}\right)}-\tan \left(\frac{\pi x}{2}\right)\right) \mathrm{d} r .
\end{aligned}
$$

Now we will show that $F$ satisfies the following properties:

$$
\begin{gathered}
F(0)=0, \\
F^{\prime}(0+)<0, \\
\lim _{a \rightarrow \infty} F(a)=0, \\
F^{\prime \prime} \text { has at most one root inside }(0, \infty) .
\end{gathered}
$$


These four conditions clearly imply that $F$ is nonpositive on $(0, \infty)$, which is precisely the claim. The first property is trivial. To check (2.17), note that $F^{\prime}(0+)$ equals

$$
1-\frac{2}{\pi} \arctan \left(\frac{e^{\pi(-x-1) / 2}}{\cos \left(\frac{\pi x}{2}\right)}+\tan \left(\frac{\pi x}{2}\right)\right)-\frac{2}{\pi} \arctan \left(\frac{e^{\pi(-x+1) / 2}}{\cos \left(\frac{\pi x}{2}\right)}-\tan \left(\frac{\pi x}{2}\right)\right)
$$

and hence we must show that

$$
\left(\frac{e^{\pi(-x-1) / 2}}{\cos \left(\frac{\pi x}{2}\right)}+\tan \left(\frac{\pi x}{2}\right)\right)\left(\frac{e^{\pi(-x+1) / 2}}{\cos \left(\frac{\pi x}{2}\right)}-\tan \left(\frac{\pi x}{2}\right)\right)>1 .
$$

This can be rewritten in the form

$$
f(x):=\exp \left(\frac{\pi}{2} x\right)-\exp \left(-\frac{\pi}{2} x\right)-\left[\exp \left(\frac{\pi}{2}\right)-\exp \left(-\frac{\pi}{2}\right)\right] \sin \left(\frac{\pi}{2} x\right)<0
$$

and follows from $f(0)=f(1)=0$ and the fact that $f$ is strictly convex on $[0,1]$. Indeed,

$$
f^{\prime \prime}(x)=\frac{\pi^{2}}{4}\left\{\exp \left(\frac{\pi}{2} x\right)-\exp \left(-\frac{\pi}{2} x\right)+\left[\exp \left(\frac{\pi}{2}\right)-\exp \left(-\frac{\pi}{2}\right)\right] \sin \left(\frac{\pi}{2} x\right)\right\}>0 .
$$

We turn to (2.18). Observe that if $a \rightarrow \infty$, then $\Phi_{a}^{\prime}(t) \rightarrow t$ for any fixed $t$. Since $\left|\Phi_{a}^{\prime}(t)\right| \leq|t|$ for all $t$, we obtain, by Lebesgue's dominated convergence theorem,

$$
\begin{aligned}
\lim _{a \rightarrow \infty} F(a) & =\frac{1}{2} \int_{\mathbb{R}} \frac{e^{\pi r / 2} \cos \left(\frac{\pi x}{2}\right)(r+x+1)}{e^{\pi r}+2 e^{\pi r / 2} \sin \left(\frac{\pi x}{2}\right)+1} \mathrm{~d} r+\frac{1}{2} \int_{\mathbb{R}} \frac{e^{\pi r / 2} \cos \left(\frac{\pi x}{2}\right)(r+x-1)}{e^{\pi r}+2 e^{\pi r / 2} \sin \left(\frac{\pi x}{2}\right)+1} \mathrm{~d} r \\
& =\frac{1}{2} \int_{\mathbb{R}} \frac{e^{\pi r / 2} \cos \left(\frac{\pi x}{2}\right)(x+1)}{e^{\pi r}+2 e^{\pi r / 2} \sin \left(\frac{\pi x}{2}\right)+1} \mathrm{~d} r+\frac{1}{2} \int_{\mathbb{R}} \frac{e^{\pi r / 2} \cos \left(\frac{\pi x}{2}\right)(x-1)}{e^{\pi r}+2 e^{\pi r / 2} \sin \left(\frac{\pi x}{2}\right)+1} \mathrm{~d} r .
\end{aligned}
$$

Here in the latter passage we have used the fact that the functions

$$
r \mapsto \frac{e^{\pi r / 2} \cos \left(\frac{\pi x}{2}\right)}{e^{\pi r} \pm 2 e^{\pi r / 2} \sin \left(\frac{\pi x}{2}\right)+1}
$$

are even on $\mathbb{R}$. It suffices to apply (2.15) and (2.18) follows. Finally, let us look at (2.19). The equation $F^{\prime \prime}(a)=0$ is equivalent to

$$
\begin{aligned}
& {\left[\cosh \left(\frac{\pi(a-x-1)}{2}\right)+\sin \left(\frac{\pi x}{2}\right)\right]^{-1}+\left[\cosh \left(\frac{\pi(a-x+1)}{2}\right)-\sin \left(\frac{\pi x}{2}\right)\right]^{-1}} \\
& =\left[\cosh \left(\frac{\pi(a+x+1)}{2}\right)+\sin \left(\frac{\pi x}{2}\right)\right]^{-1}+\left[\cosh \left(\frac{\pi(a+x-1)}{2}\right)-\sin \left(\frac{\pi x}{2}\right)\right]^{-1} .
\end{aligned}
$$

This, after long, tedious, but rather straightforward computations can be rewritten in the equivalent form

$$
2 s^{2}-4 \sinh \left(\frac{\pi}{2}\right) \cdot \frac{\sin \left(\frac{\pi x}{2}\right)}{\sinh \left(\frac{\pi x}{2}\right)} s+\cosh (\pi x)-\cosh \pi+2 \sin ^{2}\left(\frac{\pi x}{2}\right)=0,
$$

where $s=\cosh \left(\frac{\pi a}{2}\right)>1$. However, for a fixed $x \in(0,1)$, the above equation has at most one root $s$ larger than 1 (and hence there is at most one positive $a$ for which $\left.F^{\prime \prime}(a)=0\right)$. Indeed, otherwise, by Viéte's formula, we would have

$$
\frac{1}{2}\left(\cosh (\pi x)-\cosh \pi+2 \sin ^{2}\left(\frac{\pi x}{2}\right)\right)>1,
$$

which is impossible. This completes the proof. 
Lemma 2.5. For any $0<a<\infty$ and $x \in(0,1)$ we have $U_{a y}(x,-x) \leq 0$.

Proof. The reasoning is similar to that of the previous lemma. Note that by (2.13) we have, for any fixed $x \in(0,1)$,

$F(a):=U_{a y}(x,-x)=-\int_{\mathbb{R}} A_{+}(r,-x) \Phi_{a}(r+x+1) \mathrm{d} r-\int_{\mathbb{R}} A_{-}(r,-x) \Phi_{a}(r+x-1) \mathrm{d} r$,

where

$$
A_{+}(r, y)=\frac{1+\cosh \left(\frac{\pi r}{2}\right) \sin \left(\frac{\pi y}{2}\right)}{2\left(\cosh \left(\frac{\pi r}{2}\right)+\sin \left(\frac{\pi y}{2}\right)\right)^{2}}, \quad A_{-}(r, y)=\frac{-1+\cosh \left(\frac{\pi r}{2}\right) \sin \left(\frac{\pi y}{2}\right)}{2\left(\cosh \left(\frac{\pi r}{2}\right)-\sin \left(\frac{\pi y}{2}\right)\right)^{2}} .
$$

A direct computation shows that

$$
A_{+}(r, y)=\frac{\mathrm{d}}{\mathrm{d} r}\left[-\frac{1}{\pi} \frac{\sin \left(\frac{\pi y}{2}\right)+e^{-\pi r / 2}}{\cosh \left(\frac{\pi r}{2}\right)+\sin \left(\frac{\pi y}{2}\right)}\right]
$$

and

$$
A_{-}(r, y)=\frac{\mathrm{d}}{\mathrm{d} r}\left[-\frac{1}{\pi} \frac{\sin \left(\frac{\pi y}{2}\right)-e^{-\pi r / 2}}{\cosh \left(\frac{\pi r}{2}\right)-\sin \left(\frac{\pi y}{2}\right)}\right]
$$

so

$$
\begin{aligned}
F^{\prime \prime}(a)= & \int_{-\infty}^{-a-x-1} A_{+}(r,-x) \mathrm{d} r+\int_{a-x-1}^{\infty} A_{+}(r,-x) \mathrm{d} r \\
& +\int_{-\infty}^{-a-x+1} A_{-}(r,-x) \mathrm{d} r+\int_{a-x+1}^{\infty} A_{-}(r,-x) \mathrm{d} r \\
= & \frac{1}{\pi}\left[-\frac{-\sin \left(\frac{\pi x}{2}\right)+e^{-\pi(-a-x-1) / 2}}{\cosh \left(\frac{\pi(-a-x-1)}{2}\right)-\sin \left(\frac{\pi x}{2}\right)}+\frac{-\sin \left(\frac{\pi x}{2}\right)+e^{-\pi(a-x-1) / 2}}{\cosh \left(\frac{\pi(a-x-1)}{2}\right)-\sin \left(\frac{\pi x}{2}\right)}\right. \\
& \left.\quad-\frac{-\sin \left(\frac{\pi x}{2}\right)-e^{-\pi(-a-x+1) / 2}}{\cosh \left(\frac{\pi(-a-x+1)}{2}\right)+\sin \left(\frac{\pi x}{2}\right)}+\frac{-\sin \left(\frac{\pi x}{2}\right)-e^{-\pi(a-x+1) / 2}}{\cosh \left(\frac{\pi(a-x+1)}{2}\right)+\sin \left(\frac{\pi x}{2}\right)}\right] .
\end{aligned}
$$

After some quite involved calculations one obtains that the sign of $F^{\prime \prime}$ is equal to that of

$$
Q(s)=s^{2}+2 \frac{\sinh \left(\frac{\pi}{2}\right) \sinh \left(\frac{\pi x}{2}\right)}{\sin \left(\frac{\pi y}{2}\right)} s-\cosh \frac{\pi(x+1)}{2} \cosh \frac{\pi(-x+1)}{2}+\cos ^{2}\left(\frac{\pi y}{2}\right),
$$

where $s=\cosh \left(\frac{\pi a}{2}\right)$. Now take $y=-x$. It is easy to see that there is $s_{0}=$ $s_{0}(x)>1$ such that $Q(s)<0$ for $s \in\left(1, s_{0}\right)$ and $Q(s)>0$ for $s>s_{0}$. Thus $F$ is convex on $\left(0, a_{0}\right)$ and concave on $\left(a_{0}, \infty\right)$, where $s_{0}=\cosh \frac{\pi a_{0}}{2}$. Obviously, we have $F(0+)=0$ (since $\Phi_{a}$ converges pointwise to 0 as $a \rightarrow 0$ ). In addition, by the Lebesgue dominated convergence theorem,

$$
\lim _{a \rightarrow \infty} F(a)=0
$$

see (2.11). In consequence, $F$ is negative on $(0, \infty)$. This proves the claim.

Lemma 2.6. For any $0<a<\infty$ and $x \geq 0$ we have

$$
U_{a y}(x, 0) \geq-x .
$$


Proof. Consider the function $\Psi_{a}: \mathbb{R} \rightarrow \mathbb{R}$ given by $\Psi_{a}(t)=\Phi_{\infty}(t)-\Phi_{a}(t)=$ $\frac{1}{2}(|t|-a)^{2} 1_{\{|t| \geq a\}}$ for $t \in \mathbb{R}$. This function is convex, and we have

$$
\begin{aligned}
U_{\Psi_{a} y}(x, 0) & =-\frac{\pi}{4} \int_{\mathbb{R}} \frac{e^{r \pi}}{\left(e^{r \pi}+1\right)^{2}} \Psi_{a}(r+x+1) \mathrm{d} r+\frac{\pi}{4} \int_{\mathbb{R}} \frac{e^{r \pi}}{\left(e^{r \pi}+1\right)^{2}} \Psi_{a}(r+x-1) \mathrm{d} r \\
& =-\frac{\pi}{4} \int_{0}^{\infty} \frac{e^{r \pi}}{\left(e^{r \pi}+1\right)^{2}} A_{\Psi_{a}}(a, r, x) \mathrm{d} r
\end{aligned}
$$

where

$$
A_{\Psi_{a}}(a, r, x)=\Psi_{a}(r+x+1)+\Psi_{a}(r-x-1)-\Psi_{a}(r+x-1)-\Psi_{a}(r-x+1) .
$$

Here we have used the fact that the function $r \mapsto e^{r \pi}\left(e^{r \pi}+1\right)^{-2}$ is even on $\mathbb{R}$. Note that $A_{\Psi_{a}}(a, r, x)$ is nonnegative. This follows from convexity of the function $\Psi_{a}$, the fact that the numbers $r+x-1, r-x+1$ lie between $r-x-1$ and $r+x+1$, and the equality

$$
\frac{(r+x-1)+(r-x+1)}{2}=\frac{(r-x-1)+(r+x+1)}{2} .
$$

This yields $U_{\Psi_{a} y}(x, 0) \leq 0$; however,

$$
U_{\Psi_{a}}(x, y)=U_{\infty}(x, y)-U_{a}(x, y)
$$

for $x \in \mathbb{R}$ and $y \in(-1,1)$. It suffices to note that $U_{\infty y}(x, 0)=-x$, by virtue of (2.11).

Now we are ready to show the properties (2.4) and (2.5) for the functions $U_{a}$.

Lemma 2.7. For any $a \in[0, \infty]$ the function $U_{a}$ satisfies (2.4).

Proof. Observe that for any $(x, y) \in \mathbb{R}^{2}$ we have

$$
\lim _{a \rightarrow \infty} U_{a}(x, y)=U_{\infty}(x, y) \text { and } \lim _{a \rightarrow 0} \frac{1}{a} U_{a}(x, y)=U_{0}(x, y),
$$

so it is enough to prove the lemma for finite and strictly positive $a$. This is straightforward. By symmetry (see Lemma 2.3), it suffices to establish the estimate for $x \geq 0$. By (2.12) and Lemma 2.3 again, we have $U_{a x}(-x,-x) \geq 0$, which, together with the convexity of $U_{a}(\cdot,-x)$, implies $U_{a x}(x,-x) \geq 0$. Combining this with Lemma 2.5yields that the function $x \mapsto U_{a x}(x,-x)$ is nondecreasing on $[0,1]$. Clearly, it is also nondecreasing on $[1, \infty): U_{a x}(x,-x)=\Phi(2 x)$ for $x$ lying in this interval. Thus, the lemma follows.

Lemma 2.8. For any $a \in[0, \infty]$ the function $U_{a}$ satisfies (2.5).

Proof. Clearly, it suffices to establish the inequality on the strip $S$. Arguing as in the previous lemma, we see that we may restrict ourselves to $a \in(0, \infty)$. For the sake of convenience and clarity of the exposition, we have decided to split the proof into a few intermediate parts.

Step 1. The lower bound. This is straightforward: the function $(x, y) \mapsto \Phi_{a}(x-y)$ is subharmonic on $S$ and agrees with $U_{a}$ at the boundary $\partial S$.

Step 2. The upper bound: a reduction. For any $x \geq 0$ and $y \in(-1,1)$, we have

$$
\left|U_{a x}(x, y)\right|<\frac{1}{\pi} \int_{\mathbb{R}} \frac{\cos \left(\frac{\pi}{2} y\right)\left|\Phi_{a}^{\prime}\left(\frac{2}{\pi} \log |s|+\operatorname{sgn} s+x\right)\right|}{\left(\sin \left(\frac{\pi}{2} y\right)+s\right)^{2}+\cos ^{2}\left(\frac{\pi}{2} y\right)} \mathrm{d} s<a .
$$


In consequence, the function $x \mapsto U_{a}(x, y)-\Phi_{a}(x-y)$ is increasing on $(-\infty, y-a]$ and decreasing on $[y+a, \infty)$. Thus it suffices to prove the majorization for $x \in$ $(y-a, y+a)$, and for these $x$ and $y$ the inequality takes the form

$$
U_{a}(x, y)-\frac{1}{2}|x-y|^{2} \leq U_{a}(0,0) .
$$

We will show this for all $x \in \mathbb{R}$ and $y \in(-1,1)$. By symmetry (Lemma 2.3), we may restrict ourselves to nonnegative $x$. Denote the left-hand side of (2.21) by $\psi(x, y)$.

Step 3. The proof of (2.21) for $x \geq 0, y \in(-1,0]$. Lemma 2.3 implies $\psi_{x}(0,0)=0$ and, by Lemma 2.2. the function $\psi(\cdot, 0)$ is concave. Thus, $\psi(x, 0) \leq \psi(0,0)$, which is precisely (2.21) with $y=0$. Now fix a positive $x$ and note that $\psi(x, \cdot)$ is concave: $\psi_{y y}(x, y)=U_{a y y}(x, y)-1=-U_{a x x}(x, y)-1<0$ in view of Lemma 2.2. However, by (2.20), $\psi_{y}(x, 0) \geq 0$, so $\psi(x, y) \leq \psi(x, 0) \leq \psi(0,0)$ for $y \in(-1,0)$.

Step 4. The proof of (2.21) for $0 \leq x \leq y<1$. First note that (2.21) is valid if $x=y$, since the function $x \mapsto \psi(x, x)$ is nonincreasing on $(0,1)$. To see this, note that we have $U_{a x}(x, x) \leq 0$ and $U_{a y}(x, x) \leq 0$ : the first estimate is precisely (2.12), while the second follows from Lemma 2.5 and the fact that $U_{a}(x, \cdot)$ is concave on $(-1,1)$. To deal with $y>x$, simply observe that for a fixed $x \in(0,1)$, the function $\psi(x, \cdot)$ is decreasing on $(x, 1]$.

Step 5. The proof of (2.21) for $x \geq y$. By Lemma 2.2 for a fixed $y \in(0,1)$, the function $\psi(\cdot, y)$ is concave. Furthermore, $\psi_{x}(y, y)<0$ in view of (2.12). This completes the proof.

The lemma below is the final step in the proof of (1.5).

Lemma 2.9. Let $\Phi \in \mathcal{C}$. Then $U_{\Phi}$ satisfies (2.4) and (2.5).

Proof. All we need is to represent $\Phi$ as a "mixture" of elements of $\mathcal{K}$. Let $\mu$ be the unique nonnegative measure on $\mathbb{R}_{+}$satisfying $\mu((a, b])=\Phi_{+}^{\prime \prime}(a)-\Phi_{+}^{\prime \prime}(b)$ for all $0 \leq a<b$. Here $\Phi_{+}^{\prime \prime}$ stands for the right-hand derivative of the concave function $\Phi^{\prime}$. Integration by parts gives that

$$
\begin{aligned}
\Phi(x) & =\int_{0}^{\infty} \Phi_{a}(x) \mu(\mathrm{d} a)+\Phi^{\prime}(0) x+\frac{\Phi_{+}^{\prime \prime}(\infty)}{2} x^{2} \\
& =\int_{0}^{\infty} \Phi_{a}(x) \mu(\mathrm{d} a)+\Phi^{\prime}(0) \Phi_{0}(x)+\frac{\Phi_{+}^{\prime \prime}(\infty)}{2} \Phi_{\infty}(x)
\end{aligned}
$$

for any $x \geq 0$. Thus the claim follows from Fubini's theorem.

Before we proceed to the applications of the above martingale inequality, let us make an important observation here.

Remark 2.10. As already mentioned, the above approach enables us to derive sharp weak type $(p, p)$ bounds for orthogonal martingales in the case when $1 \leq p \leq 2$. A natural question arises: what are the corresponding optimal constants if $p>2$ ? In particular, does the inequality

$$
\mathbb{P}\left(Y^{*} \geq 1\right) \leq\left(\int_{\mathbb{R}} \frac{|s+1|^{p}}{\cosh \left(\frac{\pi s}{2}\right)} \mathrm{d} s\right)^{-1}\|X-Y\|_{p}^{p}
$$


also hold true for $p>2$ ? Unfortunately, the answer is no. The reason is that for $p>2$ the function $\Phi(t)=|t|^{p}$ does not satisfy the property (2.5). To see this, recall an elementary bound

$$
|a+b|^{p}+|a-b|^{p} \geq 2|a|^{p}+2|b|^{p}, \quad a, b \in \mathbb{R},
$$

with strict inequality if $a b \neq 0$. It yields

$$
\begin{aligned}
U_{\Phi}(x, 0) & =\frac{U_{\Phi}(x, 0)+U_{\Phi}(-x, 0)}{2} \\
& =\frac{1}{2 \pi} \int_{\mathbb{R}} \frac{\left|\frac{2}{\pi} \log \right| s|+\operatorname{sgn} s+x|^{p}+\left|\frac{2}{\pi} \log \right| s|+\operatorname{sgn} s-x|^{p}}{s^{2}+1} \mathrm{~d} s \\
& >\frac{1}{\pi} \int_{\mathbb{R}} \frac{\left|\frac{2}{\pi} \log \right| s|+\operatorname{sgn} s|^{p}+|x|^{p}}{s^{2}+1} \mathrm{~d} s=U_{\Phi}(0,0)+\Phi(x) .
\end{aligned}
$$

It is not difficult to show that this implies that the best constant in the martingale weak type inequality is strictly larger than

$$
\left(\int_{\mathbb{R}} \frac{|s+1|^{p}}{\cosh \left(\frac{\pi s}{2}\right)} \mathrm{d} s\right)^{-1 / p}
$$

if $p>2$. Let us briefly explain this. For a fixed $p>2$, there is $\varepsilon>0$ for which the set

$$
C=\left\{(x, y) \in S: U_{\Phi}(x, y)>U_{\Phi}(0,0)+\Phi(x-y)+\varepsilon\right\}
$$

is nonempty. Let $B=\left(B^{(1)}, B^{(2)}\right)$ be a two-dimensional Brownian motion starting from $(0,0)$ and introduce the stopping time $\tau=\inf \left\{t: B_{t} \in C \cup \partial S\right\}$. Let $X_{t}=B_{\tau \wedge t}^{(1)}$ and $Y_{t}=B_{\tau \wedge t}^{(2)}$ for $t \geq 0$. Then $X$ and $Y$ are orthogonal, since $B^{(1)}$ and $B^{(2)}$ are independent. Moreover, $[X, X]_{t}=[B, B]_{\tau \wedge t}=\tau \wedge t=[Y, Y]_{t}$, so $Y$ is differentially subordinate to $X$. On the other hand, using Itô's formula, $\mathbb{E} U_{\Phi}\left(X_{t}, Y_{t}\right)=U_{\Phi}(0,0)$ for any $t \geq 0$. Since $\tau$ is exponentially integrable, we may let $t \rightarrow \infty$ and obtain the equality

$$
\mathbb{E} U_{\Phi}\left(X_{\infty}, Y_{\infty}\right)=\mathbb{E} U_{\Phi}\left(B_{\tau}^{(1)}, B_{\tau}^{(2)}\right)=U_{\Phi}(0,0) .
$$

Since $U_{\Phi}\left(B_{\tau}\right)=\left(U_{\Phi}(0,0)+\varepsilon\right) 1_{\left\{B_{\tau}^{(2)}<1\right\}}+\Phi\left(B_{\tau}^{(1)}-B_{\tau}^{(2)}\right)$, this can be rewritten in the form

$$
U_{\Phi}(0,0)=\left(U_{\Phi}(0,0)+\varepsilon\right) \mathbb{P}\left(B_{\tau}^{(2)}<1\right)+\mathbb{E} \Phi\left(B_{\tau}^{(1)}-B_{\tau}^{(2)}\right)
$$

or

$$
\left(\int_{\mathbb{R}} \frac{|s+1|^{p}}{\cosh \left(\frac{\pi s}{2}\right)} \mathrm{d} s\right) \mathbb{P}\left(Y^{*} \geq 1\right)=\varepsilon \mathbb{P}\left(Y^{*}<1\right)+\sup _{t} \mathbb{E} \Phi\left(X_{t}-Y_{t}\right) .
$$

But $C$ has an empty intersection with a certain neighborhood of $(0,0)$; this implies that $\tau>0$ almost surely and the three terms in the above equality are nonzero. This shows that (2.22) fails to hold.

\section{WEAK TYPE INEQUALity FOR Hilbert TRANSFORM}

3.1. The periodic case. Let $D$ denote the unit disc of $\mathbb{R}^{2}$. Suppose that $u$ is a harmonic function on $D$ and let $v=\mathcal{H}^{\mathbb{T}} u$ be its conjugate. Here, as usual, we identify a harmonic function on $D$ with its radial limit on the unit circle $\mathbb{T}=\left\{e^{i \theta}\right.$ : $\theta \in(-\pi, \pi]\} \simeq(-\pi, \pi]$. We have that $u, v$ satisfy Cauchy-Riemann equations and $v(0,0)=0$. Let $B$ be a two-dimensional Brownian motion starting from $(0,0)$ and 
let $\tau=\inf \left\{t:\left|B_{t}\right| \geq 1\right\}$ be the first exit time of $B$ from $D$. Then the processes $X=\left(X_{t}\right)_{t \geq 0}, Y=\left(Y_{t}\right)_{t \geq 0}$ given by

$$
\begin{aligned}
& X_{t}=u\left(B_{\tau \wedge t}\right)+v\left(B_{\tau \wedge t}\right)=u(0,0)+\int_{0+}^{t} \nabla u\left(B_{\tau \wedge s}\right)+\nabla v\left(B_{\tau \wedge s}\right) d B_{s}, \\
& Y_{t}=-u\left(B_{\tau \wedge t}\right)+v\left(B_{\tau \wedge t}\right)=-u(0,0)+\int_{0+}^{t} \nabla u\left(B_{\tau \wedge s}\right)+\nabla v\left(B_{\tau \wedge s}\right) d B_{s},
\end{aligned}
$$

are martingales. Furthermore, we have

$$
\begin{aligned}
{[X, Y]_{t} } & =-u(0,0)^{2}+\int_{0+}^{t}-\left|\nabla u\left(B_{\tau \wedge s}\right)\right|^{2}+\left|\nabla v\left(B_{\tau \wedge s}\right)\right|^{2} \mathrm{~d} s \\
& =-u(0,0)^{2},
\end{aligned}
$$

where the latter comes from Cauchy-Riemann equations. Thus, $X$ and $Y$ are orthogonal. Similarly, we compute that for any $t \geq 0$,

$$
[X, X]_{t}=u(0,0)^{2}+\int_{0+}^{t}\left|\nabla u\left(B_{\tau \wedge s}\right)\right|^{2}+\left|\nabla v\left(B_{\tau \wedge s}\right)\right|^{2} \mathrm{~d} s=[Y, Y]_{t},
$$

so $Y$ is differentially subordinate to $X$. Moreover, we have $v(0,0)=0$, which implies that $X_{0}=-Y_{0}$. Since $B_{\tau}$ is distributed uniformly on $\mathbb{T}$, we may write, by (1.5),

$$
\begin{aligned}
\frac{1}{2 \pi}\left|\left\{\theta \in(-\pi, \pi]:\left|\left(I-\mathcal{H}^{\mathbb{T}}\right) u\left(e^{i \theta}\right)\right| \geq 1\right\}\right| & \leq \frac{1}{2 \pi}\left|\left\{\theta \in(-\pi, \pi]:\left|(u-v)\left(e^{i \theta}\right)\right| \geq 1\right\}\right| \\
& =\mathbb{P}\left(\left|Y_{\infty}\right| \geq 1\right) \\
& \leq K_{\Phi} \sup _{t} \mathbb{E} \Phi\left(X_{t}-Y_{t}\right) \\
& =K_{\Phi} \int_{-\pi}^{\pi} \Phi\left(2\left|u\left(e^{i \theta}\right)\right|\right) \frac{\mathrm{d} \theta}{2 \pi} .
\end{aligned}
$$

This completes the proof of (1.2).

3.2. The nonperiodic case. To deduce the estimates for the Hilbert transform on the line, we use a standard argument known as "blowing up the circle", which is due to Zygmund ([13, Chapter XVI, Theorem 3.8). Let $f$ be a locally integrable function on $\mathbb{R}$, satisfying

$$
\int_{\mathbb{R}} \Phi(2 f(x)) \mathrm{d} x<\infty .
$$

Note that in particular this implies $\int_{\mathbb{R}}|f(x)| \mathrm{d} x<\infty$. For a given positive integer $n$ and $x \in \mathbb{R}$, put

$$
g_{n}(x)=\frac{1}{2 \pi n} \int_{-\pi n}^{\pi n} f(t) \cot \frac{x-t}{2 n} \mathrm{~d} t .
$$

As shown in [13, we have $g_{n} \rightarrow \mathcal{H}^{\mathbb{R}} f$ almost everywhere as $n \rightarrow \infty$. On the other hand, the function

$$
x \mapsto g_{n}(n x)=\frac{1}{\pi} \int_{-\pi}^{\pi} f(n t) \cot \frac{x-t}{2} \mathrm{~d} t
$$


is precisely the periodic Hilbert transform of the function $x \mapsto f(n x),|x| \leq \pi$. Therefore,

$$
\begin{aligned}
\left|\left\{x \in(-\pi n, \pi n]:\left|f(x)-g_{n}(x)\right| \geq 1\right\}\right| & =n\left|\left\{|x| \in(-\pi, \pi]:\left|\left(I-\mathcal{H}^{\mathbb{T}}\right) f(n x)\right| \geq 1\right\}\right| \\
& \leq n K_{\Phi} \int_{-\pi}^{\pi} \Phi(2 f(n x)) \mathrm{d} x \\
& =K_{\Phi} \int_{-\pi n}^{\pi n} \Phi(2 f(x)) \mathrm{d} x \\
& \leq K_{\Phi} \int_{\mathbb{R}} \Phi(2 f(x)) \mathrm{d} x .
\end{aligned}
$$

Now let $n \rightarrow \infty$ to obtain

$$
\left|\left\{x \in \mathbb{R}:\left|\left(I-\mathcal{H}^{\mathbb{R}}\right) f(x)\right|>1\right\}\right| \leq K_{\Phi} \int_{\mathbb{R}} \Phi(2 f(x)) \mathrm{d} x .
$$

To obtain the stronger version of this estimate with a nonstrict inequality on the left-hand side, recall that $\Phi^{\prime}$ is concave on $(0, \infty)$; this is one of the assumptions imposed in the definition of $\mathcal{C}$. In consequence, for any $t>0$ we have $2 \Phi^{\prime}(t) \geq$ $\Phi^{\prime}(2 t)+\Phi^{\prime}(0+)$, so integrating this from 0 to $x>0$ gives

$$
\Phi(2 x) \leq 4 \Phi(x)+2 \Phi^{\prime}(0+) x .
$$

Now apply the inequality above to $f /(1-\varepsilon)$, where $0<\varepsilon<1 / 2$. We get

$$
\left|\left\{x \in \mathbb{R}:\left|\left(I-\mathcal{H}^{\mathbb{R}}\right) f(x)\right|>1-\varepsilon\right\}\right| \leq K_{\Phi} \int_{\mathbb{R}} \Phi\left(\frac{2 f(x)}{1-\varepsilon}\right) \mathrm{d} x .
$$

By (3.1) and (3.2), the right-hand side is finite; thus, if we let $\varepsilon \rightarrow 0$ and use Lebesgue's dominated convergence theorem, we get (1.3).

Finally, observe that the weak type estimate for $I-\mathcal{H}^{\mathbb{R}_{+}}$follows immediately from the inequality we have just established. Indeed, given a locally integrable function $f$ on $\mathbb{R}_{+}$, it suffices to extend it to $\tilde{f}: \mathbb{R} \rightarrow \mathbb{R}$ by setting $\tilde{f}(x)=0$ for $x \leq 0$ and note that $\mathcal{H}^{\mathbb{R}}+f=\mathcal{H}^{\mathbb{R}} \tilde{f}$,

$$
\left|\left\{x \in \mathbb{R}_{+}:\left|\left(I-\mathcal{H}^{\mathbb{R}_{+}}\right) f(x)\right| \geq 1\right\}\right| \leq\left|\left\{x \in \mathbb{R}:\left|\left(I-\mathcal{H}^{\mathbb{R}}\right) \tilde{f}(x)\right| \geq 1\right\}\right| .
$$

3.3. Sharpness. We will prove that the constant $K_{\Phi}$ is the best possible in the weak type inequality for $I-H^{\mathbb{R}_{+}}$. This will clearly yield the optimality of this constant also in the periodic and the martingale setting. Let $G: D \cap H \rightarrow H$ be defined by $G(z)=-(1-z)^{2} /(4 z)$. Then $G$ is a conformal mapping, and hence so is its inverse $L$. Consider another conformal map $F$ from the unit disc $D$ onto the strip $S$, given by

$$
F(z)=\frac{2}{\pi} \log \left[\frac{i z-1}{z-i}\right]+i
$$

Let us list some properties of the above functions, which will be needed below. First, observe that $L$ maps $[0,1]$ onto $\left\{e^{i \theta}: 0 \leq \theta \leq \pi\right\}$. To be more specific, for $x \in[0,1]$ we have

$$
L(x)=e^{i \theta} \text {, where } \theta \in(0, \pi) \text { is uniquely determined by } x=\sin ^{2}(\theta / 2) .
$$

Moreover,

$$
L \operatorname{maps} \mathbb{R} \backslash[0,1] \text { onto }(-1,1) \text {. }
$$


For example, for negative $x$,

$$
L(x)=1-2 x-2 \sqrt{x^{2}-x} .
$$

Concerning $F$, we have that

$$
F \text { maps the unit circle onto the boundary of } S
$$

and

$$
F \text { maps }(-1,1) \text { onto }\{a i: a \in(-1,1)\} \text {. }
$$

Now we exhibit a family of examples for which the constant $K_{\Phi}$ is asymptotically attained. For any positive integer $n$, let $V_{n}(z)=F\left(L^{2 n}(z-n)\right), z \in H$, and

$$
f_{n}(x)=\frac{1}{2}\left[-\operatorname{Re} V_{n}(x)+\operatorname{Im} V_{n}(x)\right], \quad x \geq 0 .
$$

We have

$$
\left(I-\mathcal{H}^{\mathbb{R}_{+}}\right) f_{n}(x)=\left(I-\mathcal{H}^{\mathbb{R}}\right) f_{n}(x)+\left(\mathcal{H}^{\mathbb{R}}-\mathcal{H}^{\mathbb{R}_{+}}\right) f_{n}(x) .
$$

Let us analyse the two terms on the right. Since $V_{n}$ is conformal, we have $\operatorname{Im} V_{n}=$ $\mathcal{H}^{\mathbb{R}} \operatorname{Re} V_{n}$ and $\operatorname{Re} V_{n}=-\mathcal{H}^{\mathbb{R}} \operatorname{Im} V_{n}$ when restricted to $\mathbb{R}$, so

$$
\left(I-\mathcal{H}^{\mathbb{R}}\right) f_{n}(x)=f_{n}(x)-\frac{1}{2}\left[-\operatorname{Im} V_{n}(x)-\operatorname{Re} V_{n}(x)\right]=\operatorname{Im} V_{n}(x) .
$$

However, (3.3) and (3.6) imply that $V_{n}$ satisfies $\left|\operatorname{Im} V_{n}(x)\right|=1$ for $x \in[n, n+1]$. On the other hand, by (3.4) and (3.7), we see that $\operatorname{Re} V_{n}(t)=0$ if $t \notin[n, n+1]$ and

$$
\left(\mathcal{H}^{\mathbb{R}}-\mathcal{H}^{\mathbb{R}_{+}}\right) f(x)=\frac{1}{\pi} \int_{-\infty}^{0} \frac{f(t)}{x-t} \mathrm{~d} t=\frac{1}{2 \pi} \int_{-\infty}^{0} \frac{\operatorname{Im} V_{n}(t)}{x-t} \mathrm{~d} t .
$$

The next observation is that, for $z \in(-1,1)$, we have an elementary bound

$$
|F(z)|=\left|\frac{2}{\pi} \log \left(-\frac{z+i}{z-i}\right)\right| \leq 2|z| .
$$

Therefore, by (3.5),

$$
\begin{aligned}
\left|\left(\mathcal{H}^{\mathbb{R}}-\mathcal{H}^{\mathbb{R}}+\right) f_{n}(x)\right| & \leq \frac{1}{2 \pi} \int_{-\infty}^{0} \frac{\left|\operatorname{Im} F\left(L^{2 n}(t-n)\right)\right|}{x-t} \mathrm{~d} t \\
& \leq \frac{1}{2 \pi} \int_{-\infty}^{0} \frac{\left|2 L^{2 n}(t-n)\right|}{x-t} \mathrm{~d} t \leq \frac{1}{2 \pi} \int_{-\infty}^{0} \frac{|2 L(t-n)|}{x-t} \mathrm{~d} t \rightarrow 0,
\end{aligned}
$$

as $n \rightarrow \infty$. Thus we have shown that if $x \in[n, n+1]$, then the first term on the right-hand side of (3.8) is equal to \pm 1 and the second is arbitrarily small if $n$ is sufficiently large. Thus for any $\varepsilon>0$,

$$
\left|\left\{x \in \mathbb{R}:\left|\left(I-\mathcal{H}^{\mathbb{R}_{+}}\right) f_{n}(x)\right| \geq 1-\varepsilon\right\}\right| \geq 1-\varepsilon,
$$

for large $n$. Now, let us study the limit behavior of the right-hand side of (1.4). As we have already observed, $\operatorname{Re}_{n}(x)=0$ for $x \notin[n, n+1]$, which implies $f_{n}(x)=\operatorname{Im} V_{n}(x)$ for these $x$ and hence

$$
\int_{\mathbb{R}_{+}} \Phi\left(2\left|f_{n}(x)\right|\right) \mathrm{d} x=\int_{\mathbb{R}_{+} \backslash[n, n+1]} \Phi\left(\left|\operatorname{Im} V_{n}(x)\right|\right) \mathrm{d} x+\int_{n}^{n+1} \Phi\left(2\left|f_{n}(x)\right|\right) \mathrm{d} x .
$$


By the very definition of $V_{n}$ and the inequality (3.9), the first term on the right can be dealt with as follows,

$$
\int_{0}^{n} \Phi\left(\left|\operatorname{Im} V_{n}(x)\right|\right) \mathrm{d} x=\int_{-n}^{0} \Phi\left(\left|\operatorname{Im} F\left(L^{2 n}(x)\right)\right|\right) \mathrm{d} x \leq \int_{-\infty}^{0} \Phi\left(\left|2 L^{2 n}(x)\right|\right) \mathrm{d} x,
$$

and the latter integral tends to 0 as $n \rightarrow \infty$. This is a consequence of Lebesgue's dominated convergence theorem: indeed, $L^{2 n}(x) \downarrow 0$ as $n \rightarrow \infty$ and $x \mapsto \Phi\left(\left|2 L^{2}(x)\right|\right)$ is integrable on $(-\infty, 0]$. Similarly, one shows that

$$
\lim _{n \rightarrow \infty} \int_{n+1}^{\infty} \Phi\left(\left|\operatorname{Im} V_{n}(x)\right|\right) \mathrm{d} x=0 .
$$

Furthermore,

$$
\int_{n}^{n+1} \Phi\left(2\left|f_{n}(x)\right|\right) \mathrm{d} x=\int_{0}^{1} \Phi\left(-\operatorname{Re} F\left(L^{2 n}(x)\right)+\operatorname{Im} F\left(L^{2 n}(x)\right)\right) \mathrm{d} x .
$$

Now, by (3.3),

$$
\begin{aligned}
\int_{n}^{n+1} \Phi\left(2\left|f_{n}(x)\right|\right) \mathrm{d} x & =\frac{1}{2} \int_{0}^{\pi} \Phi\left(-\operatorname{Re} F\left(e^{2 n i \theta}\right)+\operatorname{Im} F\left(e^{2 n i \theta}\right)\right) \sin \theta \mathrm{d} \theta \\
& =\frac{1}{2} \int_{0}^{2 n \pi} \Phi\left(-\operatorname{Re} F\left(e^{i \theta}\right)+\operatorname{Im} F\left(e^{i \theta}\right)\right) \sin \left(\frac{\theta}{2 n}\right) \frac{\mathrm{d} \theta}{2 n} \\
& =\frac{1}{2} \int_{0}^{2 \pi} \Phi\left(-\operatorname{Re} F\left(e^{i \theta}\right)+\operatorname{Im} F\left(e^{i \theta}\right)\right) \sum_{k=0}^{n-1} \sin \left(\frac{k \pi}{n}+\frac{\theta}{2 n}\right) \frac{\mathrm{d} \theta}{2 n} \\
& =\frac{1}{2} \int_{0}^{2 \pi} \Phi\left(-\operatorname{Re} F\left(e^{i \theta}\right)+\operatorname{Im} F\left(e^{i \theta}\right)\right) \frac{\cos \left(\frac{\theta-\pi}{2 n}\right)}{2 n \sin \left(\frac{\pi}{2 n}\right)} \mathrm{d} \theta \\
& \stackrel{n \rightarrow \infty}{\longrightarrow} \frac{1}{2 \pi} \int_{0}^{2 \pi} \Phi\left(-\operatorname{Re} F\left(e^{i \theta}\right)+\operatorname{Im} F\left(e^{i \theta}\right)\right) \mathrm{d} \theta \\
& =\frac{1}{2 \pi}\left[\int_{0}^{\infty} \Phi\left(\frac{2}{\pi} \log \left(\frac{\sin \theta}{1-\cos \theta}\right)+1\right) d \theta\right. \\
& =\frac{1}{\pi}\left[\int_{0}^{\infty} \frac{\left(\left|\frac{2}{\pi} \log t+1\right|\right)}{t^{2}+1} \mathrm{~d} t+\int_{0}^{\infty} \frac{\Phi\left(\left|\frac{2}{\pi} \log t-1\right|\right)}{t^{2}+1} \mathrm{~d} t\right] \\
& =K_{\Phi}^{-1} .
\end{aligned}
$$

Combining this with (3.10) we see that the constant $K_{\Phi}$ is indeed the best possible. This completes the proof.

\section{WEAK TYPE INEQUALITY FOR RE-EXPANSION OPERATOR}

Recall the sine and cosine Fourier transforms $\mathcal{F}_{s}, \mathcal{F}_{c}$ and the Laplace transform $\mathcal{L}$ on the positive half-line. Let us also introduce the complex Fourier transform $\mathcal{F}$ on the closed upper half-plane $\bar{H}$, given by

$$
\mathcal{F} f(x, y)=\mathcal{F} f(z)=\sqrt{\frac{2}{\pi}} \int_{\mathbb{R}_{+}} f(t) e^{i z t} \mathrm{~d} t .
$$


Clearly, it suffices to prove the inequality (1.1) for $f \in C_{0}^{\infty}\left(\mathbb{R}_{+}\right)$. The substitution $g=\mathcal{F}_{c} f$ transforms this estimate into

$$
\left|\left\{x \in \mathbb{R}_{+}:\left|\mathcal{F}_{c} g(x)-\mathcal{F}_{s} g(x)\right| \geq 1\right\}\right| \leq K_{\Phi} \int_{\mathbb{R}_{+}} \Phi\left(2 \mathcal{F}_{c} g(x)\right) \mathrm{d} x .
$$

The Fourier transform satisfies

$$
|\mathcal{F} g(z)| \leq \frac{c}{1+|z|}, \quad\left|(\mathcal{F} g)^{\prime}(z)\right| \leq \frac{c}{1+|z|^{2}}
$$

for all $z \in H$ and some absolute constant $c$ depending only on $g$. Put

$$
\begin{aligned}
& u(x, y)=u_{g}(x, y)=\operatorname{Re} \mathcal{F} g(x, y)=\sqrt{\frac{2}{\pi}} \int_{\mathbb{R}_{+}} g(t) \cos t x e^{-y t} \mathrm{~d} t, \\
& v(x, y)=v_{g}(x, y)=\operatorname{Im} \mathcal{F} g(x, y)=\sqrt{\frac{2}{\pi}} \int_{\mathbb{R}_{+}} g(t) \sin t x e^{-y t} \mathrm{~d} t
\end{aligned}
$$

and observe that we have, for all $x, y \in \mathbb{R}_{+}$,

$$
\begin{array}{ll}
u(x, 0)=\mathcal{F}_{c} g(x), & v(x, 0)=\mathcal{F}_{s} g(x), \\
u(0, y)=\mathcal{L} g(y), & v(0, y)=0
\end{array}
$$

and

$$
u_{x}(0, y)=0, \quad v_{x}(0, y)=-(\mathcal{L} g)^{\prime}(y) .
$$

Now we are ready to turn to the announced weak type estimate for re-expansion operator.

4.1. Proof of (1.1). Arguing as in the proof of (1.5), it suffices to establish the estimate for the elements of the class $\mathcal{K}$. So, fix $a \in[0, \infty]$ and consider the corresponding function $\Phi_{a}$. Our argumentation will be based, as in the probabilistic setting, on the properties of the special function $U_{a}$.

The first step is to approximate $U_{a}$ by a sufficiently smooth function. Below, we will need the existence of the partial derivatives of the second order. To do this, observe that the function $x \mapsto U_{a x}(x,-x)+U_{a y}(x,-x)$ is strictly increasing on $[0,1)$; indeed, its derivative in the interior of this interval equals $2 U_{a x x}(x,-x)>0$. Furthermore, by Lemma 2.3. this function vanishes at 0 . Thus $U_{a x}(1 / 2,-1 / 2)+$ $U_{a y}(1 / 2,-1 / 2)>0$ and, in consequence, if $\delta \in(0,1 / 2)$ is sufficiently small, then for any point $(r, s)$ from the unit ball of $\mathbb{R}^{2}$ we have

$$
U_{a x}(1 / 2-\delta r,-1 / 2-\delta s)+U_{a y}(1 / 2-\delta r,-1 / 2-\delta s)>0 .
$$

Fix such a $\delta$. Let $h: \mathbb{R}^{2} \rightarrow[0, \infty)$ be a $C^{\infty}$ function, supported on the unit ball of $\mathbb{R}^{2}$ and satisfying $\int_{\mathbb{R}^{2}} h=1$ and $h(r, s)=h(-r,-s)$ for all $r, s \in \mathbb{R}$. Define $U_{a}^{(\delta)}: \mathbb{R}^{2} \rightarrow \mathbb{R}$ by the convolution

$$
U_{a}^{(\delta)}(x, y)=\int_{\mathbb{R}^{2}} U_{a}(x-\delta r, y-\delta s) h(r, s) \mathrm{d} r \mathrm{~d} s .
$$

Since $U_{a}$ is continuous, an integration by parts gives

$$
\begin{aligned}
& U_{a x}^{(\delta)}(x, y)=\int_{\mathbb{R} \times \mathbb{R}} U_{a x}(x-\delta r, y-\delta s) h(r, s) \mathrm{d} r \mathrm{~d} s, \\
& U_{a y}^{(\delta)}(x, y)=\int_{\mathbb{R} \times \mathbb{R}} U_{a y}(x-\delta r, y-\delta s) h(r, s) \mathrm{d} r \mathrm{~d} s .
\end{aligned}
$$


Now, for a fixed $g$, recall $u=u_{g}, v=v_{g}$ given by (4.3) and introduce $G: H \rightarrow \mathbb{R}$ by

$$
G(x, y)=U_{a}^{(\delta)}(u(x, y)+v(x, y),-u(x, y)+v(x, y))-U^{(\delta)}(0,0) .
$$

Applying Green's formula for the region $D_{R}=\left\{(x, y) \in \mathbb{R}^{2}: x>R^{-1}, y>\right.$ $\left.R^{-1}, x^{2}+y^{2}<R^{2}\right\}, R>1$, we obtain

$$
\iint_{D_{R}} y \Delta G(x, y) \mathrm{d} x \mathrm{~d} y=\oint_{\partial D_{R}} G(x, y)-y G_{y}(x, y) \mathrm{d} x+y G_{x}(x, y) \mathrm{d} y .
$$

Since $U_{a}$ is subharmonic on $\mathbb{R}^{2}$, so are $U_{a}^{(\delta)}$ and $G$. Thus the double integral above is nonnegative. Now let $R \rightarrow \infty$ and use (4.2). Then the integral over the arc $\left\{(x, y): x>0, y>0, x^{2}+y^{2}=R^{2}\right\}$ tends to 0 and we get

$$
0 \leq \int_{0}^{\infty} G(x, 0) \mathrm{d} x-\int_{0}^{\infty} y G_{x}(0, y) \mathrm{d} y .
$$

To deal with the second integral on the right, note that

$$
\begin{aligned}
G_{x}(0, y)= & U_{a x}^{(\delta)}(u(0, y)+v(0, y),-u(0, y)+v(0, y)) \cdot\left(u_{x}(0, y)+v_{x}(0, y)\right) \\
& +U_{a y}^{(\delta)}(u(x, y)+v(x, y),-u(x, y)+v(x, y)) \cdot\left(-u_{x}(0, y)+v_{x}(0, y)\right) \\
= & -\left[U_{a x}^{(\delta)}(\mathcal{L} g(y),-\mathcal{L} g(y))+U_{a y}^{(\delta)}(\mathcal{L} g(y),-\mathcal{L} g(y))\right](\mathcal{L} g)^{\prime}(y) .
\end{aligned}
$$

Therefore, integrating by parts yields

$$
\int_{0}^{\infty} y G_{x}(0, y) \mathrm{d} y=\int_{0}^{\infty} J^{\delta}(\mathcal{L} g(y)) \mathrm{d} y
$$

where

$$
J^{\delta}(x)=\int_{0}^{x} U_{a x}^{(\delta)}(t,-t)+U_{a y}^{(\delta)}(t,-t) \mathrm{d} t
$$

for $x \in \mathbb{R}$. We will show that $J^{\delta} \geq 0$. By symmetry of $U_{a}$ and $h$, it suffices to prove this on $[0, \infty)$. Note that for any $b \in \mathbb{R}$, the function $r \mapsto U_{a x}(r+b,-r)+$ $U_{a y}(r+b,-r)$ is strictly increasing on $[0,1]$. Therefore, if $x \leq 1 / 2$, then

$$
U_{a x}(x-\delta r,-x-\delta s)+U_{a y}(x-\delta r,-x-\delta s) \geq U_{a x}(-\delta r,-\delta s)+U_{a y}(-\delta r,-\delta s)
$$

and, by (4.5), $J^{\delta}(x) \geq J^{\delta}(0)=0$. On the other hand, if $x \geq 1 / 2$ and $x \neq 1-\delta s$, then

$$
U_{a x}(x-\delta r,-x-\delta s)+U_{a y}(x-\delta r,-x-\delta s) \geq 0 .
$$

Indeed, if $-x-\delta s<-1$, then both sides are equal: if $-x-\delta s>-1$, then the left-hand side exceeds $U_{a x}(1 / 2-\delta r,-1 / 2-\delta s)+U_{a y}(1 / 2-\delta r,-1 / 2-\delta s)$, which is positive by virtue of (4.4). Thus, by (4.5), $\left(J^{\delta}\right)^{\prime}(x) \geq 0$ and $J^{\delta}$ is a nonnegative function. Plugging this into (4.6) yields $\int_{0}^{\infty} G(x, 0) \mathrm{d} x \geq 0$, or

$$
\int_{0}^{\infty} U_{a}^{(\delta)}\left(\mathcal{F}_{c} g(x)+\mathcal{F}_{s} g(x),-\mathcal{F}_{c} g(x)+\mathcal{F}_{s} g(x)\right)-U_{a}^{(\delta)}(0,0) \mathrm{d} x \geq 0 .
$$

However, by (2.5), we have

$$
\begin{aligned}
U_{a}^{(\delta)}(x, y) & \leq \int_{\mathbb{R}^{2}}\left[U_{a}(0,0) 1_{\{|y-\delta s|<1\}}+\Phi_{a}(x-y-\delta(r-s))\right] g(r, s) \mathrm{d} r \mathrm{~d} s \\
& \leq U_{a}(0,0) 1_{\{|y|<1+\delta\}}+\Phi_{a}(x-y)+a \int_{\mathbb{R}^{2}} \delta|r-s| h(r, s) \mathrm{d} r \mathrm{~d} s,
\end{aligned}
$$


where in the second passage we have exploited the mean value property together with the fact that $\left|\Phi_{a}^{\prime}\right| \leq a$. Therefore

$$
\begin{aligned}
U_{a}(0,0) \mid & \left\{x \geq 0:\left|\left(\mathcal{F}_{c}-\mathcal{F}_{s}\right) g(x)\right| \geq 1+\delta\right\} \mid \\
\leq & \int_{\mathbb{R}_{+}} \Phi_{a}\left(2\left|\mathcal{F}_{c} g(x)\right|\right) \mathrm{d} x+a \delta \int_{\mathbb{R}^{2}}|r-s| h(r, s) \mathrm{d} r \mathrm{~d} s+U_{a}(0,0)-U_{a}^{(\delta)}(0,0)
\end{aligned}
$$

and letting $\delta \rightarrow 0$ yields

$$
\left|\left\{x \geq 0:\left|\left(\mathcal{F}_{c}-\mathcal{F}_{s}\right) g(x)\right|>1\right\}\right| \leq K_{\Phi_{a}}^{-1} \int_{\mathbb{R}_{+}} \Phi_{a}\left(2\left|\mathcal{F}_{c} g(x)\right|\right) \mathrm{d} x .
$$

Applying this inequality to $g /(1-\varepsilon)$ for $0<\varepsilon<1 / 2$ and letting $\varepsilon \rightarrow 0$ strengthens this bound to the form

$$
\left|\left\{x \geq 0:\left|\left(\mathcal{F}_{c}-\mathcal{F}_{s}\right) g(x)\right| \geq 1\right\}\right| \leq K_{\Phi_{a}}^{-1} \int_{\mathbb{R}_{+}} \Phi_{a}\left(2\left|\mathcal{F}_{c} g(x)\right|\right) \mathrm{d} x .
$$

The proof is complete.

4.2. Optimality of the constant. Fix $\varepsilon>0$ and let $f$ be a function on $\mathbb{R}_{+}$such that

$$
\left|\left\{x \geq 0:\left|\left(I-\mathcal{H}^{\mathbb{R}_{+}}\right) f(x)\right| \geq 1-\varepsilon\right\}\right| \geq 1-\varepsilon
$$

and

$$
\int_{\mathbb{R}_{+}} \Phi(2|f(x)|) \mathrm{d} x \leq K_{\Phi}^{-1}+\varepsilon .
$$

Such a function exists by the reasoning presented in the previous section. For any $s>0$, let $f^{(s)}$ be given by

$$
f^{(s)}(x)=f(x-s) 1_{\{x \geq s\}}, \quad x \geq 0 .
$$

We have that

$$
\begin{aligned}
\mid\{x \geq 0: \mid & \left.(I-\Pi) f^{(s)}(x) \mid \geq 1-2 \varepsilon\right\} \mid \\
& =\mid\left\{x \geq 0:\left|\left(I-\mathcal{H}^{\mathbb{R}_{+}}-\mathcal{H}_{1}\right) f^{(s)}(x) \geq 1-2 \varepsilon\right|\right. \\
& \geq\left|\left\{x \in \mathbb{R}:\left|\left(I-\mathcal{H}^{\mathbb{R}_{+}}\right) f^{(s)}(x)\right| \geq 1-\varepsilon\right\}\right|-\left|\left\{x \geq 0:\left|\mathcal{H}_{1} f^{(s)}(x)\right|>\varepsilon\right\}\right| \\
& =\left|\left\{x \in \mathbb{R}:\left|\left(I-\mathcal{H}^{\mathbb{R}_{+}}\right) f(x)\right| \geq 1-\varepsilon\right\}\right|-\left|\left\{x \geq 0:\left|\mathcal{H}_{1} f^{(s)}(x)\right|>\varepsilon\right\}\right| \\
& \geq 1-\varepsilon-\frac{\left\|\mathcal{H}_{1} f^{(s)}\right\|_{1}}{\varepsilon} \geq 1-2 \varepsilon,
\end{aligned}
$$

provided $s$ is sufficiently large. This follows from the equality $\mathcal{H}_{1} f^{(s)}(x)=\mathcal{H}_{1} f(x+$ $s$ ) for any $x \geq 0$. On the other hand,

$$
\int_{\mathbb{R}_{+}} \Phi\left(2\left|f^{(s)}(x)\right|\right) \mathrm{d} x=\int_{\mathbb{R}_{+}} \Phi(2|f(x)|) \mathrm{d} x .
$$

This shows that the constant $K_{\Phi}$ is indeed the best possible. 


\section{REFERENCES}

[1] R. Bañuelos and G. Wang, Sharp inequalities for martingales with applications to the Beurling-Ahlfors and Riesz transformations, Duke Math. J. 80 (1995), 575-600. MR1370109 (96k:60108)

[2] M. S. Birman, Re-expansion operators as objects of spectral analysis, in: Linear and Complex Analysis Problem Book, Lecture Notes in Math. 1043, Springer, 1984, 130-134.

[3] B. Davis, On the weak type $(1,1)$ inequality for conjugate functions, Proc. Amer. Math. Soc. 44 (1974),307-311. MR0348381 (50:879)

[4] C. Dellacherie and P.-A. Meyer, Probabilities and potential B: Theory of martingales, North Holland, Amsterdam, 1982. MR745449 (85e:60001)

[5] E. M. Essén, A superharmonic proof of the M. Riesz conjugate function theorem, Ark. Mat. 22 (1984), pp. 241-249. MR765412 (86c:30068)

[6] T. W. Gamelin, Uniform Algebras and Jensen Measures, London Math. Soc. Lecture Notes Series, Vol. 32, Cambridge Univ. Press, Cambridge/New York, 1978. MR.521440 (81a:46058)

[7] I. Gohberg and N. Krupnik, Norm of the Hilbert transformation in the $L_{p}$ space, Funct. Anal. Pril. 2 (1968), 91-92 [in Russian]; English transl. in Funct. Anal. Appl. 2 (1968), 180-181.

[8] B. Hollenbeck, N. J. Kalton and I. E. Verbitsky, Best constants for some operators associated with the Fourier and Hilbert transforms, Studia Math. 157 (2003), 237-278. MR1980300 (2004b:41035)

[9] B. Hollenbeck and I. E. Verbitsky, Best Constants for the Riesz Projection, J. Funct. Anal. 175 (2000), 370-392. MR1780482 (2001i:42010)

[10] P. Janakiraman, Best weak-type ( $p, p)$ constants, $1 \leq p \leq 2$ for orthogonal harmonic functions and martingales, Illinois J. Math. 48 No. 3 (2004), 909-921. MR2114258 (2005m:60088)

[11] S. K. Pichorides, On the best values of the constants in the theorems of M. Riesz, Zygmund and Kolmogorov, Studia Math. 44 (1972), 165-179. MR0312140 (47:702)

[12] G. Wang, Differential subordination and strong differential subordination for continuous time martingales and related sharp inequalities, Ann. Probab. 23 (1995), 522-551. MR.1334160 (96b:60120)

[13] A. Zygmund, Trigonometric series Vol. 2, Cambride University Press, London, 1968. MR0236587 (38:4882)

Department of Mathematics, Informatics and Mechanics, University of Warsaw, BaNACHA 2, 02-097 WARSAW, POLAND

E-mail address: ados@mimuw.edu.pl 